\title{
Non-Monotonic Inference Properties for Assumption-Based Argumentation
}

\author{
Kristijonas Čyras and Francesca Toni \\ Imperial College London, UK \\ $\{\mathrm{k}$. cyras13,ft\}@imperial.ac.uk
}

\begin{abstract}
Cumulative Transitivity and Cautious Monotonicity are widely considered as important properties of non-monotonic inference and equally as regards to information change. We propose three novel formulations of each of these properties for Assumption-Based Argumentation (ABA) an established structured argumentation formalism, and investigate these properties under a variety of ABA semantics.
\end{abstract}

Keywords: Assumption-Based Argumentation · Non-Monotonic Inference $\cdot$ Argumentation dynamics

\section{Introduction}

In the 1980s, several non-monotonic reasoning formalisms were proposed (see [2] for an overview). Systemic investigations into aspects of Cautious Monotonicity and Cumulative Transitivity of non-monotonic inference followed (e.g. [22, 23]). Those works also contribute to the well-studied area of analysing non-monotonic reasoning with respect to information change (c.f. [27]).

Since the early 1990s, argumentation (as overviewed in [26]) has emerged as a generic framework for non-monotonic reasoning, admitting existing nonmonotonic reasoning formalisms as instances $[7,15]$. Recently, some forms of structured argumentation (see [5] for an overview) have been investigated in terms of non-monotonic inference (c.f. Sect. 4). Contributing to this area of research, we here analyse a well-established structured argumentation formalismAssumption-Based Argumentation (ABA) (see [28] for a tutorial) - against the non-monotonic inference properties of Cumulative Transitivity and Cautious Monotonicity in the spirit of $[22,23]$. Since ABA is an instance of a well-known structured argumentation framework ASPIC+ (see [24] for a tutorial), this work is potentially applicable to a wider array of argumentation systems.

The original properties were defined with respect to non-monotonic entailment. Yet, ABA (as well as a significant portion of other structured argumentation formalisms) is defined in terms of extensions (e.g. sets of arguments). We thus first reformulate the properties to be applicable to extension-based nonmonotonic reasoning formalisms (but see e.g. $[10,14]$ for different approaches). The essential idea is to characterize what happens to extensions when a certain change in knowledge occurs. The following will serve as an abstract pattern 
for producing the concrete instances of the properties (from now on, CUT and MON stand for Cumulative Transitivity and Cautious Monotonicity, respectively):

Let $\mathcal{K}$ be a knowledge base. Suppose that an 'entity' $\psi$ 'belongs' to an extension $E$ of $\mathcal{K}$, and let $E^{\prime}$ be an extension of the knowledge base $\mathcal{K}^{\prime}$, which is obtained by 'adding' $\psi$ to $\mathcal{K}$. Then

$$
\text { CUT : } E \text { 'contains' } E^{\prime} ; \quad \text { MON : } E^{\prime} \text { 'contains' } E \text {. }
$$

These properties concern what happens when a conclusion that is reachedwhich could have been already present as a hard fact, or inferred defeasibly - is added to the knowledge base and reasoned with anew. Arguably, there are many ways to interpret both properties, e.g. as checking that accepting a conclusion does not yield overwhelming changes in reasoning. One of our contributions is to provide three instantiations of both CUT and MON applicable to ABA. We will also discuss some possible interpretations of those instantiations.

The abstract formulation above, aiming to be universal, is informal: notions like 'entity' act as placeholders for alternative formal concepts (e.g. conclusion of an argument); 'containment' need not be understood in set-theoretic terms. For $\mathrm{ABA}$, we will provide rigorously defined instances of the abstract formulation.

To ease the intuition behind the properties, consider the following illustration.

Example 1. Three prospective academic partners-Alphons, Benedict and Dalton-invite you to dine at a new restaurant. On the eve of the dinner it turns out that you will have to sit in pairs at two separate tables. In a playful manner, your associates compete for your company: both Benedict and Dalton claim that Alphons is antisocial, while Alphons retorts that Benedict is back-stabbing. Somewhat puzzled, you casually inquire about the restaurant. Benedict says it is a gourmet place. You recall that Dalton is a disagreeable person over fancy food. It is high time to decide, so what will be the verdict? The reasoning may unfold as follows. Benedict defends himself against Alphons by insisting that the latter is antisocial. Meanwhile, Alphons has nothing against his attacker Dalton. The latter is not a good option, assuming that Benedict is right about gourmet food. No more hesitating, and you decide to go for Benedict.

Now, how would the information that you are really in a gourmet place change your reasoning, if at all? One can argue that, knowing as a matter of fact it is a gourmet restaurant immediately discards Dalton as an option. So if Dalton is out of consideration, then Alphons is attacked only by Benedict, and in turn attacks him back. Thus both Benedict and Alphons are acceptable choices. In terms of non-monotonic inference, CUT insists you should not draw any new conclusions, while MON demands not to lose previous inferences. Sticking to your first choice would satisfy both requirements, whereas choosing Alphons over Benedict would violate both properties, indicating a revision of your previous decision.

In this work we investigate how ABA (background in Sect. 2) behaves when employed to formalize this sort of situations. In particular, in Sect. 3 we provide three instantiations of each of CUT and MON, and analyse their satisfaction under six extension-based ABA semantics. After discussing related work (Sect. 4), we conclude in Sect. 5. 


\section{Background}

In this section, we provide background on $\mathrm{ABA}$, following [28].

An ABA framework is a tuple $(\mathcal{L}, \mathcal{R}, \mathcal{A},-)$ consisting of the following elements. $(\mathcal{L}, \mathcal{R})$ is a deductive system with a language $\mathcal{L}$ and a set $\mathcal{R}$ of rules: rules in $\mathcal{R}$ are assumed to be of the form $\varphi_{0} \leftarrow \varphi_{1}, \ldots, \varphi_{m}$ with $m \geq 0$ and $\varphi_{i} \in \mathcal{L}$ for $i \in\{0, \ldots, m\} ; \varphi_{0}$ is referred to as the head, and $\varphi_{1}, \ldots, \varphi_{m}$ is referred to as the body of the rule; if $m=0$, then the rule is said to have an empty body and we write it as $\varphi_{0} \leftarrow \top$. The set $\mathcal{A} \subseteq \mathcal{L}$ is non-empty, referred to as assumptions. The so called contrary mapping $-: \mathcal{A} \rightarrow \mathcal{L}$ is a total function and for $\alpha \in \mathcal{A}$, the $\mathcal{L}$-formula $\bar{\alpha}$ is referred to as the contrary of $\alpha$.

As usual in the literature, we restrict the discussion to so called flat ABA frameworks, where no assumption $\alpha \in \mathcal{A}$ can be the head of any rule from $\mathcal{R}$.

A deduction for $\varphi \in \mathcal{L}$ supported by $S \subseteq \mathcal{L}$ and $R \subseteq \mathcal{R}$, denoted by $S \vdash^{R} \varphi$, is a finite tree with the root labeled by $\varphi$, leaves labeled by $\top$ or elements from $S$, the children of non-leaf nodes $\psi$ labeled by the elements of the body of some rule from $\mathcal{R}$ with the head $\psi$, and $R$ being the set of all such rules. An argument $A$ with conclusion $\varphi \in \mathcal{L}$ and support $A \subseteq \mathcal{A}$, written as $\mathrm{A}: A \vdash \varphi$, is a deduction for $\varphi$ supported by $A$ and some $R \subseteq \mathcal{R}$. We say that $\mathrm{A}^{\prime}: A^{\prime} \vdash \varphi^{\prime}$ attacks A : $A \vdash \varphi$ (on some $\alpha \in A$ ) just in case $\varphi^{\prime}$ is the contrary $\bar{\alpha}$ of some $\alpha \in \bar{A}$.

Given an ABA framework $(\mathcal{L}, \mathcal{R}, \mathcal{A},-)$, we denote the set of constructible arguments by Args, the attack relation by $\rightsquigarrow$, and the corresponding argument framework by $($ Args,$\rightsquigarrow)$. For a set $S \subseteq$ Args, we say that: $S$ attacks an argument $\mathrm{A}^{\prime}$, written $S \rightsquigarrow \mathrm{A}^{\prime}$, if some A $\in S$ attacks $\mathrm{A}^{\prime} ; S$ attacks a set $S^{\prime} \subseteq$ Args of arguments, written $S \rightsquigarrow S^{\prime}$, if $S$ attacks some $\mathrm{A}^{\prime} \in S^{\prime} ; S$ is conflict-free if $S \not \leftrightarrow S$; and $S$ defends $\mathrm{A} \in$ Args if for each $\mathrm{A}^{\prime} \rightsquigarrow \mathrm{A}$ we have $S \rightsquigarrow \mathrm{A}^{\prime}$. For an argument A, let $C n(\mathrm{~A})$ be the conclusion of $\mathrm{A}$ and $\operatorname{asm}(\mathrm{A})$ the support of $\mathrm{A}$. We extend this notation so that for a set $S \subseteq$ Args of arguments, $C n(S)=\{C n(\mathrm{~A}): \mathrm{A} \in S\}$ and $\operatorname{asm}(S)=\{\alpha \in \mathcal{A}: \alpha \in \operatorname{asm}(\mathrm{A}), \mathrm{A} \in S\}$.

ABA semantics are as follows. A set $E \subseteq A r g s$, also called an extension (of $(\mathcal{L}, \mathcal{R}, \mathcal{A},-)$ or $($ Args, $\rightsquigarrow)$ ), is: admissible, if $E$ is conflict-free and defends all A $\in E$; preferred, if $E$ is $\subseteq$-maximally admissible; sceptically preferred, if $E$ is the intersection of all the preferred extensions; complete, if $E$ is admissible and contains all arguments it defends; grounded, if $E$ is $\subseteq$-minimally complete; stable, if $E$ is admissible and $E \rightsquigarrow \mathrm{A}$ for all $\mathrm{A} \in$ Args $\backslash E$; and ideal, if $E$ is $\subseteq$-maximal such that $E$ is admissible and contained in all the preferred extensions.

Grounded, sceptically preferred and ideal semantics fall into the category of sceptical reasoning, whereby conclusions are drawn from a unique extension. Meanwhile stable, preferred and complete semantics represent credulous reasoning, in that multiple conflicting extensions can be present.

We also recall (c.f. [15]) that the grounded extension $G$ of any $(\mathcal{L}, \mathcal{R}, \mathcal{A},-)$ always exists and is unique, and can be constructed inductively as $G=\bigcup_{i>0} G_{i}$, where $G_{0}$ is the set of arguments that are not attacked at all, and for every $i \geq 0$, $G_{i+1}$ is the set of arguments that are defended by $G_{i}$.

To simplify proofs of our results, we restrict to finite argument frameworks, as is common in literature. 


\section{Inference Properties for ABA}

In this section we formulate and analyse non-monotonic inference properties regarding ABA. There will be three different settings of instantiations of CUT and MON. Each property will also have a strong and a weak version. The strong properties will quantify over all extensions, indicating the necessity to preserve the previously accepted conclusions after a change in information. Meanwhile, the weak properties, by quantifying existentially over extensions, will insist on the possibility, rather than necessity. When referring to a property, we will have in mind its strong version, unless specified otherwise.

Throughout this section (except for the examples) we use the following notation. We assume as given a fixed, but otherwise arbitrary (flat) ABA framework $\mathcal{F}=(\mathcal{L}, \mathcal{R}, \mathcal{A},-)$, and its corresponding argument framework (Args, $)$. To instantiate the abstract formulations of CUT and MON given in the Introduction, we replace a knowledge base $\mathcal{K}$ with $\mathcal{F}$, fix an argumentation semantics $\sigma$ and let $E$ be an extension of $\mathcal{F}$ under $\sigma$. An 'entity' $\psi$ will come from the set $C n(E)$ of conclusions of $E$. By default, the knowledge base $\mathcal{K}^{\prime}$ will be represented by $\mathcal{F}^{\prime}$ - the ABA framework obtained by 'adding' (to be formalized) $\psi$ to $\mathcal{F}$, with the corresponding argument framework $\left(\right.$ Args $\left.^{\prime}, \rightsquigarrow^{\prime}\right)$. Still further, $E^{\prime}$ will denote an extension of $\mathcal{F}^{\prime}$ according to the same fixed semantics $\sigma$. To avoid trivialities, we consider cases only where under a particular semantics $\sigma$, each of $\mathcal{F}$ and $\mathcal{F}^{\prime}$ has at least one extension- $E$ and $E^{\prime}$ respectively.

\subsection{Strict Cumulative Transitivity and Cautious Monotonicity}

We now rigorously formulate the first type of properties for ABA. First, given $\psi \in C n(E) \backslash \mathcal{A}$, define $\mathcal{F}^{\prime}=\left(\mathcal{L}, \mathcal{R} \cup\{\psi \leftarrow \top\}, \mathcal{A},-{ }^{-}\right)$. The following then are the first concrete instances of non-monotonic inference properties that we consider.

STRONG STRICT CUT : For all extensions $E^{\prime}$ of $\mathcal{F}^{\prime}$ we have $C n\left(E^{\prime}\right) \subseteq C n(E)$;

WEAK STRICT CUT : $\quad$ There is an extension $E^{\prime}$ of $\mathcal{F}^{\prime}$ with $C n\left(E^{\prime}\right) \subseteq C n(E)$;

STRONG STRICT MON : For all extensions $E^{\prime}$ of $\mathcal{F}^{\prime}$ we have $C n(E) \subseteq C n\left(E^{\prime}\right)$;

WEAK STRICT MON : There is an extension $E^{\prime}$ of $\mathcal{F}^{\prime}$ with $C n(E) \subseteq C n\left(E^{\prime}\right)$.

STRICT CUT and STRICT MON concern what happens when a conclusion (not itself an assumption) is reached and then considered as a fact (i.e. a rule with empty body) to reason again. The conclusion may be learned as an objective truth, e.g. verifying that you are in a gourmet restaurant. In essence, STRICT properties regard strengthening of information and what effect it has on different ABA semantics in terms of extensions. A reasoner employing ABA semantics can utilize these properties to anticipate its behaviour regarding changes that strengthen knowledge.

The following remarks are in place. First, satisfaction of a strong property will always imply satisfaction of the corresponding weak property. Second, under sceptical semantics, weak and strong formulations actually coincide, because 
the extension is unique. Finally, if a strong property is violated under stable semantics, then it fails under both preferred and complete semantics, because stable extensions are preferred, and preferred extensions are complete [7].

Our first result shows that grounded semantics fulfills (the strong versions of) both CUT and MON in the STRICT setting.

Proposition 2. Grounded semantics satisfies both STRICT CUT and STRICT MON.

Proof. Let $G$ be the grounded extension of $\mathcal{F}$. If $G=\emptyset$, then $\mathcal{F}^{\prime}=\mathcal{F}$, so the properties are trivially satisfied. Otherwise, pick $\psi \in C n(G) \backslash \mathcal{A}$ and let $\mathrm{B}_{1}: B_{1} \vdash \psi, \ldots, \mathrm{B}_{n}: B_{n} \vdash \psi$ be all the arguments in $G$ that have conclusion $\psi$. Let $G^{\prime}$ be the grounded extension of $\mathcal{F}^{\prime}=\left(\mathcal{L}, \mathcal{R} \cup\{\psi \leftarrow \top\}, \mathcal{A},{ }^{-}\right)$.

We prove $G \subseteq G^{\prime}$ by induction on the construction of $G$.

For the basis step, let $G_{0} \subseteq G$ be the set of arguments not attacked in $\mathcal{F}$. Since clearly $C n\left(\right.$ Args $\left.^{\prime}\right)=C n(\bar{A} r g s)$, we obtain $G_{0} \subseteq G^{\prime}$.

For the inductive step, let $G_{i+1} \subseteq G$ be the set of arguments attacked in $\mathcal{F}$ but defended by $G_{i} \subseteq G$, assuming $G_{i} \subseteq G^{\prime}$ as an induction hypothesis. Suppose $\mathrm{A}^{\prime}: A^{\prime} \vdash \varphi$ attacks $G_{i+1}$ in $\mathcal{F}^{\prime}$. If $\mathrm{A}^{\prime} \in A r g s$, then $G_{i} \rightsquigarrow \mathrm{A}^{\prime}$, and so $G^{\prime} \rightsquigarrow \mathrm{A}^{\prime}$ too. Else, if $\mathrm{A}^{\prime} \notin A \mathrm{Args}$, then there is some $\mathrm{A}: A \vdash \varphi \in \operatorname{Args}$ from which $\mathrm{A}^{\prime}$ can be obtained by replacing occurrences of the deduction $B_{j} \vdash^{R_{j}} \psi$ (for some $j$ ) in $A$ with the deduction $\emptyset \vdash^{-\{\psi \leftarrow T\}} \psi$. (Such $A^{\prime}$ and $A$ are called counterpart $\operatorname{arguments}$ and satisfy $\operatorname{asm}(\mathrm{A})=\operatorname{asm}\left(\mathrm{A}^{\prime}\right) \cup B_{j}$.) We then have $\mathrm{A} \rightsquigarrow G_{i+1}$, so that $G_{i} \rightsquigarrow$ A on some $\alpha \in A \backslash B_{j}=A^{\prime}$, which yields $G^{\prime} \rightsquigarrow{ }^{\prime} \mathrm{A}^{\prime}$. In any event, $G^{\prime}$ defends $G_{i+1}$, so that $G_{i+1} \subseteq G^{\prime}$.

By induction it holds that $G_{i} \subseteq G^{\prime}$ for every $i \geq 0$, so that $G \subseteq G^{\prime}$, and hence $C n(G) \subseteq C n\left(G^{\prime}\right)$, giving STRICT MON.

For STRICT CUT, given that we already have $G \subseteq G^{\prime}$, it suffices to show that $C n\left(G^{\prime} \backslash G\right) \subseteq C n(G)$. We prove this by induction on the construction of $G^{\prime}$.

For the basis step, let $G_{0}^{\prime} \subseteq G^{\prime} \backslash G$ be the set of arguments from Args $^{\prime} \backslash$ Args unattacked in $\mathcal{F}^{\prime}$. Pick $\mathrm{A}^{\prime} \in G_{0}^{\prime}$, if any. Consider a counterpart $\mathrm{A} \in \operatorname{Args}$ with $\operatorname{asm}(\mathrm{A})=\operatorname{asm}\left(\mathrm{A}^{\prime}\right) \cup B_{j}\left(\right.$ for some $j$ ) and $C n(\mathrm{~A})=C n\left(\mathrm{~A}^{\prime}\right)$. Such an $\mathrm{A}$ can be attacked in $\mathcal{F}$ only on some $\beta \in B_{j}$, whereby $G$ defends A. Consequently, $C n\left(\mathrm{~A}^{\prime}\right) \in C n(G)$, and therefore, $C n\left(G_{0}^{\prime}\right) \subseteq C n(G)$.

For the inductive step, let $G_{i+1}^{\prime} \subseteq G^{\prime} \backslash G$ be the set of arguments attacked in $\mathcal{F}^{\prime}$ but defended by $G \cup G_{i}^{\prime}$, assuming $C n\left(G_{i}^{\prime}\right) \subseteq C n(G)$. Pick $\mathrm{A}^{\prime} \in G_{i+1}^{\prime}$, if any, and likewise consider a counterpart $\mathrm{A} \in$ Args, which can be attacked in $\mathcal{F}$ in two ways: either on some $\beta \in B_{j}$, whence $G$ defends $\mathrm{A}$ in $\mathcal{F}$; or on some $\alpha \in \operatorname{asm}(\mathrm{A}) \backslash B_{j}$, whence $\mathrm{A}^{\prime}$ is attacked in $\mathcal{F}^{\prime}$ (on $\alpha$ ), and so defended in $\mathcal{F}^{\prime}$ by $G \cup G_{i}^{\prime}$, so that $G$ defends A in $\mathcal{F}$. In any case, $\mathrm{A} \in G$, and so $C n\left(G_{i+1}^{\prime}\right) \subseteq C n(G)$.

$C n\left(G^{\prime}\right) \subseteq C n(G)$ now holds by induction, as required.

So we now know that strong, and hence weak, STRICT CUT and STRICT MON hold for grounded semantics. What is more, weak versions of both properties are satisfied under complete semantics, as we see next.

Proposition 3. Complete semantics satisfies WEAK STRICT CUT and WEAK STRICT MON. 
Proof. We prove that for each complete extension $E$ of $\mathcal{F}$, and for each conclusion $\psi \in C n(E) \backslash \mathcal{A}$, there is a complete extension $E^{\prime}$ of $\mathcal{F}^{\prime}=\left(\mathcal{L}, \mathcal{R} \cup\{\psi \leftarrow \top\}, \mathcal{A},{ }^{-}\right)$ such that $C n\left(E^{\prime}\right)=C n(E)$.

Let $E$ be a complete extension of $\mathcal{F}$ and fix $\psi \in C n(E) \backslash \mathcal{A}$. Suppose that $\mathrm{B}_{1}: B_{1} \vdash \psi, \ldots, \mathrm{B}_{n}: B_{n} \vdash \psi$ are all the arguments in $E$ with conclusion $\psi$. Now, Args ${ }^{\prime} \backslash$ Args consists of arguments $\mathrm{A}^{\prime}: A^{\prime} \vdash \varphi$ which are constructed from arguments A : $A \vdash \varphi$ in Args that use some deduction(s) of the form $\Psi \vdash^{R} \psi$, by replacing (some) such deduction(s) with $\emptyset \vdash\{\psi \leftarrow T\} \psi$. (Such $A$ and $A^{\prime}$ are said to be corresponding to each other.) Let $E^{+}$be the collection of $\mathrm{A}^{\prime} \in \mathrm{Args}^{\prime} \backslash$ Args whose corresponding $\mathrm{A}$ is in $E$. We claim that $E^{\prime}:=E \cup E^{+}$is the required complete extension of $\mathcal{F}^{\prime}$.

First, $E^{\prime}$ is conflict-free, as $C n\left(E^{+}\right) \subseteq C n(E)$. Moreover, $E^{\prime}$ defends every argument it contains: if $\mathrm{A}^{\prime} \in \operatorname{Args}^{\prime} \backslash$ Args attacks $E^{\prime}$ in $\mathcal{F}^{\prime}$, but $E^{\prime} \not \boldsymbol{w}^{\prime} \mathrm{A}^{\prime}$, then a counterpart (as in the proof of Proposition 2) argument A attacks $E$ in $\mathcal{F}$, but $E \not \rightarrow \mathrm{A}$, contradicting admissibility of $E$. Finally, for completeness, assume $E^{\prime}$ defends $\mathrm{A}^{\prime} \in A r g s^{\prime}$. If $\mathrm{A}^{\prime} \in A r g s$, then $E$ defends $\mathrm{A}^{\prime}$ in $\mathcal{F}^{\prime}$, so $\mathrm{A}^{\prime} \in E$. Else, if $\mathrm{A}^{\prime} \notin A$ rgs, then assume $\mathrm{A}^{\prime} \notin E^{+}$for a contradiction. Then a counterpart $\mathrm{A} \in$ Args is not in $E$, and so some $\mathrm{C}$ attacks $\mathrm{A}$ in $\mathcal{F}$, but $E \ngtr \rightarrow \mathrm{C}$. As $E$ defends all $\mathrm{B}_{j} \mathrm{~s}$, we have $\mathrm{C} \rightsquigarrow^{\prime} \mathrm{A}^{\prime}$, but $E^{\prime} \not \varkappa^{\prime} \mathrm{C}$ - a contradiction to $E^{\prime}$ defending $\mathrm{A}^{\prime}$. In any event, $A^{\prime} \in E^{\prime}$. Hence, $E^{\prime}$ is complete.

We can actually extend the proof above to be applicable to both preferred and stable semantics, as follows.

Proposition 4. Preferred and stable semantics satisfy WEAK STRICT CUT and WEAK STRICT MON.

Proof. We prove that for every preferred extension $E$ of $\mathcal{F}$, there is a preferred extension $E^{\prime}$ of $\mathcal{F}^{\prime}$ with $C n\left(E^{\prime}\right)=C n(E)$. Since preferred extensions are complete, it suffices to show that the corresponding complete extension $E^{\prime}=E \cup E^{+}$ (as defined in the proof of Proposition 3) is preferred in $\mathcal{F}^{\prime}$. Indeed, if $E^{\prime}$ were not $\subseteq$-maximally admissible, then some $\mathrm{A}^{\prime} \in \operatorname{Args}^{\prime} \backslash E^{\prime}$ could be added to $E^{\prime}$ without sacrificing admissibility. But then a counterpart $\mathrm{A} \in \operatorname{Args}$ (possibly $\mathrm{A}=\mathrm{A}^{\prime}$, if $\mathrm{A}^{\prime}$ does not use $\psi$ ) could be added to $E$ without losing its admissibility, whence $E$ would not be preferred in $\mathcal{F}$.

Likewise, we show that if $E$ is stable, then $E^{\prime}$ is also stable. Suppose $A^{\prime} \notin E^{\prime}$. If $\mathrm{A}^{\prime} \in A$ rgs, then $\mathrm{A}^{\prime} \notin E$, so $E \rightsquigarrow \mathrm{A}^{\prime}$, and hence $E^{\prime} \rightsquigarrow^{\prime} \mathrm{A}^{\prime}$. Else, if $\mathrm{A}^{\prime} \notin A r g s$, then a counterpart $A$ is not in $E$ and $E \rightsquigarrow A$, so that $E^{\prime} \rightsquigarrow^{\prime} A^{\prime}$ too.

Having the results above, we conclude with the following.

Corollary 5. Sceptically preferred and ideal semantics satisfy STRICT CUT.

Proof. According to Proposition 4 (on preferred extensions), the sceptically preferred (resp., ideal) extension $S^{\prime}$ (resp., $I^{\prime}$ ) of $\mathcal{F}^{\prime}$ by definition cannot contain arguments with conclusions not in $C n(S)$ (resp., $C n(I)$ ), where $S$ (resp., $I$ ) is the sceptically preferred (resp., ideal) extension of $\mathcal{F}$. 
The following formalization of our illustrative example from the Introduction reveals that neither of the (strong) properties holds under credulous reasoning. This violation is intuitive, because credulous semantics allow for multiple extensions, with not all conclusions in common.

Example 6 (STRICT CUT and STRICT MON violations). Let $\mathcal{L}=\{\alpha, \beta, \delta, a, b, d, \psi\}$, where: $\alpha, \beta, \delta$ are the assumptions of choosing Alphons, Benedict and Dalton (resp.); $a, b$ and $d$ stand for 'antisocial', 'back-stabbing' and 'disagreeable' (resp.); and $\psi$ expresses that we are in a gourmet place. So $\mathcal{A}=\{\alpha, \beta, \delta\}$, with contraries $\bar{\alpha}=a, \bar{\beta}=b, \bar{\delta}=d$. The rules $\mathcal{R}=\{b \leftarrow \alpha, a \leftarrow \delta, a \leftarrow \beta, \psi \leftarrow \beta, d \leftarrow \psi\}$ complete the formalization: e.g. $b \leftarrow \alpha$ represents Alphons's claim about Benedict; $d \leftarrow \psi$ indicates that Dalton is a disagreeable company in a gourmet place. (In further examples, both $\mathcal{L}$ and $\mathcal{A}$ will be omitted, as they are implicit from $\mathcal{R}$ and the contrary relation.) The corresponding argument framework (Args, $)$ can be represented graphically as follows (a directed edge indicates an attack):

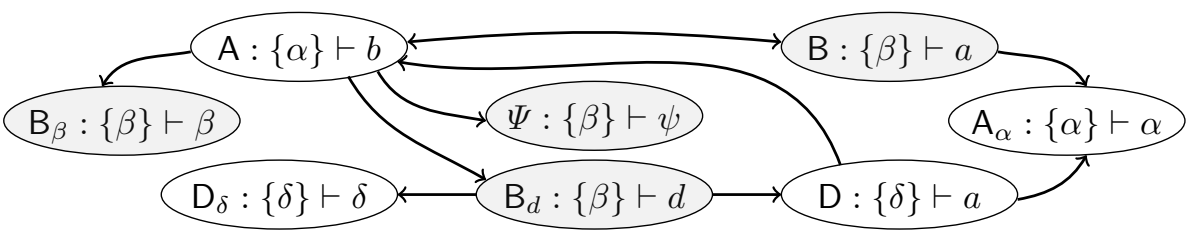

$\mathcal{F}$ has a unique preferred (also stable and ideal) extension $E=\left\{\mathrm{B}, \mathrm{B}_{\beta}, \mathrm{B}_{d}, \Psi\right\}$ (gray arguments) with $C n(E)=\{a, \beta, d, \psi\}$. Now suppose that after deciding to sit with Benedict, you check the menu and realize you are indeed in a gourmet restaurant. As knowledge changes - your belief that this is a gourmet place being strengthened-you wonder whether you would make the same decision now.

Consider thus $\mathcal{F}^{\prime}=(\mathcal{L}, \mathcal{R} \cup\{\psi \leftarrow \top\}, \mathcal{A},-)$. In Args $^{\prime}$, we get two new arguments: $\Psi^{\prime}:\{\} \vdash \psi$ and $\mathrm{B}^{\prime}:\{\} \vdash d$. While $\Psi^{\prime}$ neither attacks nor is attacked, $\mathrm{B}^{\prime}$ is unattacked but attacks both $\mathrm{D}_{\delta}$ and D. So $\left(\right.$ Args $\left.^{\prime}, \rightsquigarrow^{\prime}\right)$ has two preferred extensions (which are also stable): $E_{1}=\left\{\mathrm{B}, \mathrm{B}_{\beta}, \mathrm{B}_{d}, \mathrm{~B}^{\prime}, \Psi, \Psi^{\prime}\right\}$ (with $C n\left(E_{1}\right)=C n(E)$ ) and $E_{2}=\left\{\mathrm{A}_{\alpha}, \mathrm{A}, \mathrm{B}^{\prime}, \Psi^{\prime}\right\}$. Taking $E_{2}$ with $C n\left(E_{2}\right) \nsubseteq \subset C n(E) \nsubseteq \subset C n\left(E_{2}\right)$ yields violations of STRICT CUT and STRICT MON under credulous reasoning. We also have $C n(E) \nsubseteq C n\left(\left\{\mathrm{~B}^{\prime}, \Psi^{\prime}\right\}\right)=C n\left(E_{1} \cap E_{2}\right)$, so STRICT MON is violated under both ideal and sceptically preferred semantics.

So a reasoner using ABA could find itself in a situation where adding credulously inferred information leads to a multitude of extensions. Even if the extension to begin with is unique, as in this case, strengthening some of its conclusions can result in more than one acceptable extension. Whether or not this behaviour is desirable may depend on the application, anticipated changes in information and intended flexibility of the reasoner. For instance, one may wish for the reasoner to be credulous and try many different scenarios in order not to fixate on one particular decision. Meanwhile, sceptical semantics (except grounded) provide insurance that no new conclusions are attained-fulfill STRICT CUT, while ensuring that some are dropped (e.g. $\beta, d$ ). However, a sceptical reasoner may completely lose some previously acceptable choices (such as $\beta$ here). 
Example 6 also reveals contrast between STRICT CUT and STRICT MON under sceptically preferred and ideal semantics: adding a previously attained conclusion as a fact leaves all the original preferred extensions intact, yet allows for new ones, thus possibly shrinking their intersection.

We observe that under credulous semantics, the strong properties gain importance in settings where there is a unique credulous extension to begin with, such as in Example 6. Indeed, while the weak properties merely ask for the existence of an extension (of the framework after the knowledge change) with the same conclusions as the original one, the strong properties require all new extensions to commit to the conclusions of the original one. The two properties together essentially insist that the new framework should admit a unique extension having the same conclusions as the original extension.

The following table summarizes this subsection's results (as indicated, strong and weak versions coincide under sceptical reasoning, and for credulous semantics the status of the weak property is indicated in parentheses).

STRICT Cumulative Transitivity and Cautious Monotonicity

\begin{tabular}{|c|c|c|c|c|c|c|}
\hline Property & Grounded & Ideal & Sc. pref. & Stable & Preferred & Complete \\
\hline STRICT CUT & $\checkmark$ & $\checkmark$ & $\checkmark$ & X $(\checkmark)$ & X $(\checkmark)$ & X $(\checkmark)$ \\
\hline STRICT MON & $\checkmark$ & X & X & X $(\checkmark)$ & X $(\checkmark)$ & X $(\checkmark)$ \\
\hline
\end{tabular}

Only grounded semantics allows for safely strengthening information. However, as the grounded extension of a given ABA framework can be empty (c.f. Example 6), other semantics may be needed to make decisions. In that case, ideal and sceptically preferred semantics, for instance, guarantee that no new conclusions will be attained after strengthening information, yet some important ones may be lost: in Example 6, neither semantics allows to decide whom to dine with, because $\alpha, \beta, \delta \notin C n\left(E_{1} \cap E_{2}\right)$. Credulous semantics provide even less certainty, or more flexibility - depending on the way one intends to use it, unless one has a procedure allowing to pick the extension with the same conclusions as the extension to begin with (guaranteed by the satisfaction of the weak properties).

\subsection{Defeasible Cumulative Transitivity and Cautious Monotonicity}

We now formulate another type of variants of CUT and MON. Given $\psi \in C n(E) \backslash \mathcal{A}$, define $\mathcal{F}^{\prime}=\left(\mathcal{L} \cup\{y\}, \mathcal{R} \backslash\{r \in \mathcal{R}\right.$ : head of $r$ is $\left.\psi\}, \mathcal{A} \cup\{\psi\},{ }^{-}\right) .{ }^{1}$ Then

STRONG DEF CUT : For all extensions $E^{\prime}$ of $\mathcal{F}^{\prime}$ we have $C n\left(E^{\prime}\right) \subseteq C n(E)$;

WEAK DEF CUT : $\quad$ There is an extension $E^{\prime}$ of $\mathcal{F}^{\prime}$ with $C n\left(E^{\prime}\right) \subseteq C n(E)$;

STRONG DEF MON : For all extensions $E^{\prime}$ of $\mathcal{F}^{\prime}$ we have $C n(E) \subseteq C n\left(E^{\prime}\right)$;

WEAK DEF MON : $\quad$ There is an extension $E^{\prime}$ of $\mathcal{F}^{\prime}$ with $C n(E) \subseteq C n\left(E^{\prime}\right)$.

\footnotetext{
${ }^{1}$ The modification of the rules in $\mathcal{F}^{\prime}$ is required to preserve flatness. We also slightly abuse the notation by using - for both contrary mappings - the implicit presumption is that the original ${ }^{-}$is extended with the assignment $\bar{\psi}=y$, where $y$ is new to $\mathcal{L}$.
} 
Unlike the STRICT setting, DEF CUT and DEF MON regard situations where a previously accepted conclusion (inferred possibly defeasibly using assumptions) is converted into an assumption itself, and can afterwards be drawn only defeasibly. For instance, instead of relying on Benedict's claim about gourmet food, you may guess to begin with that you are in a gourmet place.

The same results (as in Sect. 3.1) hold in the defeasible (DEF) setting, via very similar proofs. We emphasize only the pivotal aspects.

Proposition 7. Grounded semantics satisfies both DEF CUT and DEF MON.

Proof (Sketch). By reasoning as in the proof of Proposition 2, it can be shown by induction on the construction of the grounded extension $G$ (resp. $G^{\prime}$ ) that $C n(G) \subseteq C n\left(G^{\prime}\right)$ (resp. $C n\left(G^{\prime} \backslash G\right) \subseteq C n(G)$ ) by using: corresponding arguments $\mathrm{A}^{\prime} \in$ Args $^{\prime} \backslash$ Args (with $\{\psi\} \vdash^{\emptyset} \psi$ replacing all $\Psi \vdash^{R} \psi$ ) of arguments A $\notin$ Args $^{\prime}$ that use deduction(s) $\Psi \vdash^{R} \psi$; and counterparts $C^{\prime} \in$ Args $^{\prime} \backslash$ Args (that use $\{\psi\} \vdash^{\emptyset} \psi$ instead of some fixed $B_{j} \vdash^{R_{j}} \psi$ ) of arguments $\mathrm{C} \in$ Args.

Proposition 8. Complete semantics satisfies WEAK DEF CUT and WEAK DEF MON.

Proof (Sketch). With $E$ complete for $\mathcal{F}$, using corresponding and counterpart arguments as in the proof (sketch) of Proposition 7, one shows that with $E^{+}=$ $\left\{\mathrm{A}^{\prime}\right.$ corresponding to $\left.\mathrm{A} \in E\right\}$, set $E^{\prime}:=\left(E \cap A r g s^{\prime}\right) \cup E^{+}$is complete for $\mathcal{F}^{\prime}$.

Verbatim to the proofs of Proposition 4 and Corollary 5 (resp.), we have:

Proposition 9. Preferred and stable semantics satisfy WEAK DEF CUT and MON.

Corollary 10. Sceptically preferred and ideal semantics satisfy DEF CUT.

The following example exhibits a violation of both DEF CUT and DEF MON under the remaining semantics.

Example 11 (DEF CUT and DEF MON violations. Based on Example 6). Suppose that instead of relying on Benedict about the restaurant (remove $\psi \leftarrow \beta$ ), you guess it to be a gourmet place to begin with (add $\psi$ to assumptions). Reason then according to $(\mathcal{L} \cup\{y\}, \mathcal{R} \backslash\{\psi \leftarrow \beta\}, \mathcal{A} \cup\{\psi\},-)$ (with $\bar{\psi}=y$ ), where the corresponding argument framework is as follows:

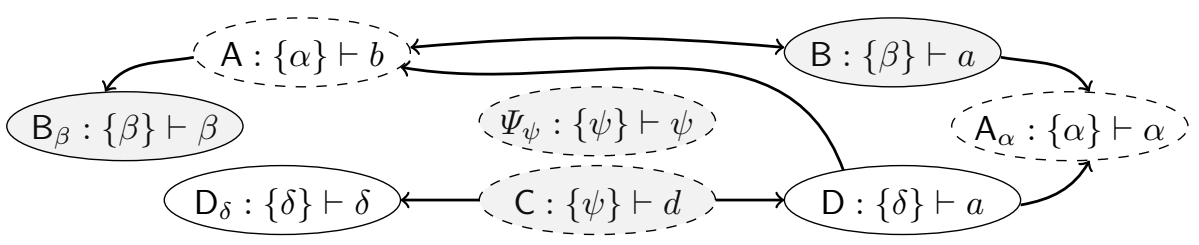

There are two preferred extensions (which are also stable): $E_{1}^{\prime}=\left\{\mathrm{B}, \mathrm{B}_{\beta}, \mathrm{C}, \Psi_{\psi}\right\}$ (gray) and $E_{2}^{\prime}=\left\{\mathrm{A}_{\alpha}, \mathrm{A}, \mathrm{C}, \Psi_{\psi}\right\}$ (dashed). The sceptically preferred (also ideal) extension is $E^{\prime}=\left\{\mathrm{C}, \Psi_{\psi}\right\}$ with $C n\left(E^{\prime}\right) \nsupseteq\{a, \beta, \psi, d\}=C n(E)$, where $E$ is as in Example 6. So DEF MON fails under both sceptically preferred and ideal semantics. DEF CUT and DEF MON fail in credulous reasoning, as $C n(E) \nsubseteq C n\left(E_{2}^{\prime}\right) \nsubseteq C n(E)$. 
Even when starting with a unique credulous extension, assuming a previously defeasibly inferred conclusion opens up space for multiple credulous extensions. This may be desirable in situations where revision of decisions based on defeasible assumptions ( $\beta$ in this case) is important. At the same time, such behaviour results into possibly losing conclusions in sceptical reasoning (except, as before, under grounded semantics). This nevertheless may be sensible, if, for instance, differentiating defeasible information is needed (e.g. $\psi$ versus $\psi \leftarrow \beta$ ).

Below is a summary of results in this subsection (using the same notational conventions as at the end of Sect. 3.1).

DEFEASIBLE Cumulative Transitivity and Cautious Monotonicity

\begin{tabular}{|c|c|c|c|c|c|c|}
\hline Property & Grounded & Ideal & Sc. pref. & Stable & Preferred & Complete \\
\hline DEF CUT & $\checkmark$ & $\checkmark$ & $\checkmark$ & X $(\checkmark)$ & X $(\checkmark)$ & X $(\checkmark)$ \\
\hline DEF MON & $\checkmark$ & X & X & X $(\checkmark)$ & X ( $\checkmark)$ & X $(\checkmark)$ \\
\hline
\end{tabular}

Again, conclusions drawn using grounded semantics can be safely turned into assumptions and inferred defeasibly instead. However, such a change would not allow for new conclusions under the other two sceptical semantics, yet could lead to a decision vacuum: neither of $\alpha, \beta, \delta$ belongs to $C n\left(E^{\prime}\right)$ in Example 11. Credulous semantics, meanwhile, allow for greater dynamicity, which could be desirable: if independently from what Benedict says a reasoner believes to be in a gourmet place and thus does not care about Dalton, then Alphons can be as likely a choice as Benedict, and so the conclusions may need revision.

Naturally, somewhat different formulations of the properties in the defeasible setting could be investigated. For example, the contrary of the new assumption $\psi$ could instead be one of the existing symbols in $\mathcal{L}$, based on the rules and contraries of the assumptions that allowed to derive $\psi$ in the first place. However, such formulations can be difficult to formulate, and equally hard to grasp. We chose the one above, readily applicable to all ABA frameworks, as the first step in our analysis. Different and more complex settings are left for future work.

\subsection{Assumption Cumulative Transitivity and Cautious Monotonicity}

Previously discussed properties focused on non-assumption conclusions. We now turn to conclusions that are assumptions, as follows. Given $\psi \in \operatorname{Cn}(E) \cap \mathcal{A}$, define $\mathcal{F}^{\prime}=\left(\mathcal{L}, \mathcal{R} \cup\{\psi \leftarrow \top\}, \mathcal{A} \backslash\{\psi\},{ }^{-}\right) .^{2}$ Then

STRONG ASM CUT : For all extensions $E^{\prime}$ of $\mathcal{F}^{\prime}$ we have $C n\left(E^{\prime}\right) \subseteq C n(E)$;

WEAK ASM CUT : $\quad$ There is an extension $E^{\prime}$ of $\mathcal{F}^{\prime}$ with $C n\left(E^{\prime}\right) \subseteq C n(E)$;

STRONG ASM MON : For all extensions $E^{\prime}$ of $\mathcal{F}^{\prime}$ we have $C n(E) \subseteq C n\left(E^{\prime}\right)$;

WEAK ASM MON : $\quad$ There is an extension $E^{\prime}$ of $\mathcal{F}^{\prime}$ with $C n(E) \subseteq C n\left(E^{\prime}\right)$.

\footnotetext{
${ }^{2}$ Again, for brevity reasons, the same symbol ${ }^{-}$is used for both contrary mappings, and in the new framework $\mathcal{F}^{\prime}$, the contrary mapping - is implicitly restricted to a diminished set of assumptions.
} 
ASM CUT and ASM MON focus on previously accepted assumptions being confirmed and made into facts to reason again. For instance, you might have guessed that you are in a gourmet restaurant, and after deciding whom to sit with you may check the menu to confirm your guess and scrutinize your decision.

As for satisfaction of the properties, the same results (as in Sect. 3.1, 3.2) hold with proofs following the same pattern, so only crucial aspects will be stressed.

Proposition 12. Grounded semantics satisfies both ASM CUT and ASM MON.

Proof (Sketch). In $\mathcal{F}^{\prime}$, deductions $\Phi \vdash^{R} \varphi$ having $\psi \in \Phi$ are replaced with $\Phi \backslash\{\psi\} \vdash^{\prime} \cup\{\psi \leftarrow \top\} \quad \varphi$, where bodies of rules from $R^{\prime} \subseteq R$ do not contain $\psi$. Arguments $\mathrm{A} \in \operatorname{Args}$ with $\psi \in \operatorname{asm}(\mathrm{A})$ have counterparts $\mathrm{A}^{\prime} \in$ Args $^{\prime}$ with $\operatorname{asm}\left(\mathrm{A}^{\prime}\right)=\operatorname{asm}(\mathrm{A}) \backslash\{\psi\}$. So $C n(G) \subseteq C n\left(G^{\prime}\right)\left(\right.$ resp. $\left.C n\left(G^{\prime}\right) \subseteq C n(G)\right)$ is shown by induction on the construction of the grounded extension $G$ (resp. $G^{\prime}$ ).

Proposition 13. Complete semantics satisfies WEAK ASM CUT and MON.

Proof (Sketch). With $E$ complete for $\mathcal{F}$, sets $E^{-}:=\{\mathrm{A} \in E: \psi \in \operatorname{asm}(\mathrm{A})\}$ and $E^{+}:=\left\{A^{\prime}\right.$ is counterpart of $\left.A \in E^{-}\right\}$, one shows that $E^{\prime}:=\left(E \backslash E^{-}\right) \cup E^{+}$ is a complete extension of $\mathcal{F}^{\prime}$.

The following two results can be obtained similarly as before.

Proposition 14. Preferred and stable semantics satisfy WEAK ASM CUT and MON.

Corollary 15. Sceptically preferred and ideal semantics satisfy ASM CUT.

Under the remaining semantics, the properties are violated.

Example 16 (ASM CUT and ASM MON violations). Consider $\mathcal{R}=\{d \leftarrow \alpha, a \leftarrow \beta$, $b \leftarrow \alpha, \delta\}$ with $\bar{\alpha}=a, \bar{\beta}=b, \bar{\delta}=d$. This yields the following (Args, $\rightsquigarrow)$ :

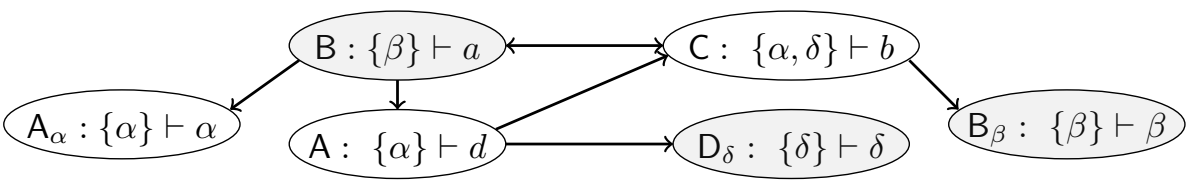

Here, $E=\left\{\mathrm{B}, \mathrm{B}_{\beta}, \mathrm{D}_{\delta}\right\}$ (gray) is a unique preferred (also stable and ideal) extension. Taking $\delta \in C n(E) \cap \mathcal{A}$ results in $\mathcal{F}^{\prime}=\left(\mathcal{L}, \mathcal{R} \cup\{\delta \leftarrow \top\}, \mathcal{A} \backslash\{\delta\},{ }^{-}\right)$in which $\mathrm{C}$ and $\mathrm{D}_{\delta}$ are replaced by their counterparts $\mathrm{C}^{\prime}:\{\alpha\} \vdash b$ and $\mathrm{D}_{\delta}^{\prime}:\{\} \vdash \delta$ :

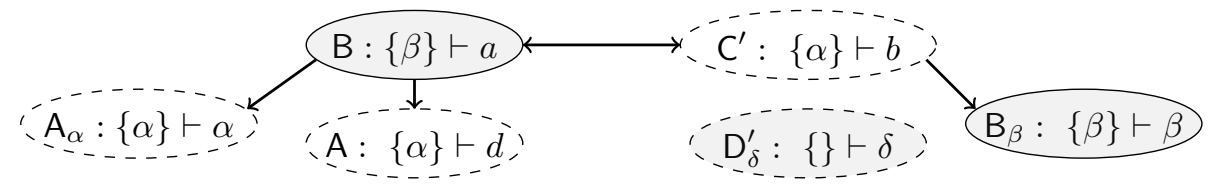

Therefore, $\mathcal{F}^{\prime}$ admits two preferred extensions: $E_{1}^{\prime}=\left\{\mathrm{B}, \mathrm{B}_{\beta}, \mathrm{D}_{\delta}^{\prime}\right\}$ (gray) and $E_{2}^{\prime}=\left\{\mathrm{A}_{\alpha}, \mathrm{C}^{\prime}, \mathrm{D}_{\delta}^{\prime}, \mathrm{A}\right\}$ (dashed) with $C n(E) \nsubseteq C n\left(E_{2}^{\prime}\right) \nsubseteq C n(E)$. The sceptically preferred and ideal extension is $E^{\prime}=\left\{\mathrm{D}_{\delta}^{\prime}\right\}$ with $C n(E) \nsubseteq C n\left(E^{\prime}\right)$. 
Compared to sceptical semantics, credulous ones are more dynamic. Here, for instance, confirming $\delta$ results in retracting $\beta$ (as well as $a$ ) under both ideal and sceptically preferred semantics. Meanwhile, the same change effectively disables the argument $A$ and leads to $B$ losing its position as the sole defender against $A$, hence enabling mutual acceptability of $\alpha$ and $\delta$, under, say, complete semantics. This allows for a possibly desirable revision of conclusions.

The following is a summary of this subsection's results (notation as before).

ASSUMPTION Cumulative Transitivity and Cautious Monotonicity

\begin{tabular}{|c|c|c|c|c|c|c|}
\hline Property & Grounded & Ideal & Sc. pref. & Stable & Preferred & Complete \\
\hline ASM CUT & $\checkmark$ & $\checkmark$ & $\checkmark$ & X $(\checkmark)$ & X $(\checkmark)$ & X $(\checkmark)$ \\
\hline ASM MON & $\checkmark$ & X & X & X $(\checkmark)$ & X $(\checkmark)$ & X $(\checkmark)$ \\
\hline
\end{tabular}

We see that confirmation of some defeasible information can lead to an increased number of options in credulous reasoning. This could be desirable if, for instance, one of the choices (like $C$ with conclusion $b$ in Example 16) depends on an assumption $(\delta)$ and is not considered acceptable to begin with ( $C$ has no defense against $A$ ), but becomes viable (via $C^{\prime}$ ) as soon as the assumption is confirmed $(\delta \leftarrow \top)$ and ceases to be questioned $\left(\mathrm{D}_{\delta}^{\prime}\right)$. Meanwhile, if confirming information widens the array of credulous choices, then a sceptical reasoner could opt for fewer-more certain - conclusions, as witnessed by the sceptical (bar grounded) semantics satisfying ASM CUT but failing ASM MON.

\section{Related Work}

The two most related works to ours are Hunter's [21] and Dung's [16]. The former investigates non-monotonic inference properties with respect to argumentclaim entailment in logic-based argumentation systems. Given various base logics, Hunter defines argument construction-mimicking entailment operators to produce claims from knowledge bases, and examines those operators against nonmonotonic inference properties (Cumulative Transitivity and Cautious Monotonicity among them). Meanwhile, Dung analyses, among other aspects of argumentation dynamics, Cumulativity (i.e. Cumulative Transitivity plus Cautious Monotonicity) of ASPIC + under stable extension semantics. The main concern there is that confirmation of some conclusions in an extension should strengthen other conclusions in that extension. To formalize this, Dung introduces two axioms - a variant of Cumulativity and another one regarding attack monotonicity. Stable extension semantics with respect to either of the main four ASPIC+ attack relations are shown not to satisfy at least one of those axioms.

Other related work falls under two broad research topics in argumentation: (i) analysing desirable properties of argumentation formalisms, and (ii) relating belief change and argumentation. Regarding (i), with the exceptions of [16] and [21], existing works on properties of argumentation disregard the issues of argumentation dynamics: for example, [11] propose rationality postulates for rulebased argumentation systems; [17] provide guidelines for argumentation-based 
practical reasoning; [20] postulate and examine properties of attack relations (and the corresponding extensions under alternative semantics) over classical logic-based argument graphs. As far as (ii) is concerned, argumentation dynamics has recently been studied with respect to Abstract Argumentation [15] and some other argumentation-based approaches to non-monotonic reasoning, such as DeLP [19] (see e.g. $[3,12,13,18]$ ). To the best of our knowledge, [16] is the only work in the direction of investigating structured, extension-based argumentation with regards to non-monotonic inference properties á la [22].

Our work differs from [16] in several aspects. First, we consider Cumulative Transitivity and Cautious Monotonicity as two separate properties, rather than one. Also, our reformulations of the properties are not restricted to one particular semantics (stable extensions), but allow for any semantics. Still further, we consider three types of information change, including strengthening (STRICT) and confirmation (ASM), and analyse their influence to argumentation processes in ABA. Finally, we do not insist that properties have to be necessarily fulfilled, but maintain that their satisfaction is conditional on applications.

\section{Conclusions}

This paper researches extension-based structured argumentation dynamics in the spirit of non-monotonic inference properties of $[22,23]$. To this end, we offer reformulations of non-monotonic inference properties in terms of extensions. Particularly, we introduce (strong and weak versions of) six properties applicable to the well-known structured argumentation formalism ABA and investigate their satisfaction under six key ABA semantics. Three pairs of properties reflect different modifications of knowledge in ABA frameworks, and each item of a pair concerns either Cumulative Transitivity (CUT) or Cautious Monotonicity (MON) of extension-based non-monotonic inference. While conceptually the three types of information change are different, we show that technically they lead to the same outcomes in the sense of a property being satisfied in either all or none of the three settings, under a particular semantics. Consequently, irrespective of the knowledge representation in $\mathrm{ABA}$ and the nature of the anticipated changes in information, one can choose semantics best suited for the application, depending on the desirable properties of the reasoner.

Credulous semantics violate the strong properties. This is expected, due to presence of choice between extensions that share conclusions. Meanwhile, the weak properties are satisfied under credulous semantics. This essentially says that ABA frameworks do not lose the extension based on which a change in knowledge occurs. As for further results on credulous reasoning, we can also identify a certain provocative aspect of our findings: even when a stable/preferred extension to begin with is unique, changing (even strengthening) information in ABA can lead to more than one stable/preferred extension afterwards (Examples $6,11,16)$. We believe that this phenomenon itself deserves further study in terms of characterization of ABA frameworks and/or semantics for which it occurs. 
In terms of sceptical reasoning, intuitively, the most sceptical (grounded) semantics satisfies all the properties. This is because grounded extensions commit to the most certain conclusions to begin with, and changing the way they are represented in ABA frameworks does not influence their (and other arguments') acceptance. Somewhat surprisingly, the other two sceptical semantics - sceptically preferred and ideal - fail MON, yet fulfill CUT. Such a behaviour is present because changes in information can increase the number of, particularly, preferred extensions, whence their intersection shrinks, resulting in violation of MON, at the same time satisfying CUT.

The results provide guidelines regarding argumentation dynamics for modeling common-sense reasoning using ABA. Due to the same property satisfaction outcomes, irrespective of knowledge representation in ABA one has a range of differently behaving semantics to choose among, contingent on the intended behaviour of the reasoner. Depending on application, one may wish to rely on the static grounded semantics to prevent overwhelming changes in reasoning, or use a much more dynamic credulous semantics to be flexible about revising decisions.

This work serves as one of the first steps towards investigating extensionbased structured argumentation dynamics. Current results cover ABA, and hence (by virtue of results in [25]) ASPIC+ without preferences, with regards to nonmonotonic inference properties CUT and MON. Future work directions include investigations of different formulations of the properties, as well as analysis of extension-based formalisms of argumentation with preferences, such as AS$\mathrm{PIC}+$, Value-Based Argumentation [4] or PAFs [1], against variants of the nonmonotonic inference properties in question. It may also be possible to use the abstract formulations of the properties to analyse other non-monotonic reasoning formalisms, such as default logic and logic programming (c.f. $[8,9]$ ), from a slightly different perspective than in the existing work (e.g. $[6,10,14])$.

\section{References}

1. Amgoud, L., Vesic, S.: Rich Preference-Based Argumentation Frameworks. Int. J. Approx. Reason. 55(2), 585-606 (2014)

2. Antoniou, G.: Nonmonotonic Reasoning. MIT Press (1997)

3. Baroni, P., Boella, G., Cerutti, F., Giacomin, M., van der Torre, L., Villata, S.: On the Input/Output Behavior of Argumentation Frameworks. Artif. Intell. 217, 144-197 (2014)

4. Bench-Capon, T.: Persuasion in Practical Argument Using Value-Based Argumentation Frameworks. J. Log. Comput. 13(3), 429-448 (2003)

5. Besnard, P., García, A., Hunter, A., Modgil, S., Prakken, H., Simari, G., Toni, F.: Introduction to Structured Argumentation. Argument Comput. 5(1), 1-4 (2014)

6. Bochman, A.: A Foundational Theory of Belief and Belief Change. Artif. Intell. 108(1-2), 309-352 (1999)

7. Bondarenko, A., Dung, P.M., Kowalski, R., Toni, F.: An Abstract, ArgumentationTheoretic Approach to Default Reasoning. Artif. Intell. 93(97), 63-101 (1997)

8. Brewka, G., Eiter, T.: Preferred Answer Sets for Extended Logic Programs. Artif. Intell. 109(1-2), 297-356 (1999) 
9. Brewka, G., Eiter, T.: Prioritizing Default Logic. In: Intellectics Comput. Log. pp. 27-45 (2000)

10. Brewka, G., Niemelä, I., Truszczynski, M.: Nonmonotonic reasoning. In: van Harmelen, F., Lifschitz, V., Bruce, P. (eds.) Handb. Knowl. Represent., pp. 239284. Elsevier (2007)

11. Caminada, M., Amgoud, L.: On the Evaluation of Argumentation Formalisms. Artif. Intell. 171(5-6), 286-310 (2007)

12. Cayrol, C., de Saint-Cyr, F.D., Lagasquie-Schiex, M.C.: Change in Abstract Argumentation Frameworks: Adding an Argument. CoRR (2014)

13. Coste-Marquis, S., Konieczny, S., Mailly, J.G., Marquis, P.: On the Revision of Argumentation Systems: Minimal Change of Arguments Status. In: KR (2014)

14. Dix, J.: A Classification Theory of Semantics of Normal Logic Programs: I. Strong Properties. Fundam. Informaticae 22(3), 227-255 (1995)

15. Dung, P.M.: On the Acceptability of Arguments and its Fundamental Role in Nonmonotonic Reasoning, Logic Programming and n-person Games. Artif. Intell. 77, 321-357 (1995)

16. Dung, P.M.: An Axiomatic Analysis of Structured Argumentation for Prioritized Default Reasoning. In: ECAI. pp. 267-272 (2014)

17. Dung, P.M., Mancarella, P., Toni, F.: Some Design Guidelines for Practical Argumentation Systems. In: COMMA. pp. 183-194 (2010)

18. Falappa, M., García, A., Kern-Isberner, G., Simari, G.: On the Evolving Relation between Belief Revision and Argumentation. Knowl. Eng. Rev. 26(01), 35-43 (2011)

19. García, A., Simari, G.: Defeasible Logic Programming: DeLP-servers, Contextual Queries, and Explanations for Answers. Argument Comput. 5(1), 63-88 (2014)

20. Gorogiannis, N., Hunter, A.: Instantiating Abstract Argumentation with Classical Logic Arguments: Postulates and Properties. Artif. Intell. 175(9-10), 1479-1497 (2011)

21. Hunter, A.: Base Logics in Argumentation. In: COMMA. pp. 275-286 (2010)

22. Kraus, S., Lehmann, D., Magidor, M.: Nonmonotonic Reasoning, Preferential Models and Cumulative Logics. Artif. Intell. 44(1-2), 167-207 (1990)

23. Makinson, D.: General Theory of Cumulative Inference. In: Reinfrank, M., de Kleer, J., Ginsberg, M.L., Sandewall, E. (eds.) Lect. Notes Comput. Sci. NonMonotonic Reason., pp. 1-18. Springer Verlag (1989)

24. Modgil, S., Prakken, H.: The ASPIC+ Framework for Structured Argumentation: A Tutorial. Argument Comput. 5(1), 31-62 (2014)

25. Prakken, H.: An Abstract Framework for Argumentation with Structured Arguments. Argument Comput. 1(2), 93-124 (2010)

26. Rahwan, I., Simari, G.: Argumentation in Artificial Intelligence. Springer (2009)

27. Rott, H.: Change, Choice and Inference: A Study of Belief Revision and Nonmonotonic Reasoning. Oxford University Press (2001)

28. Toni, F.: A Tutorial on Assumption-Based Argumentation. Argument Comput. $5(1), 89-117$ (2014) 